$\xi=-$

\title{
A rare non-auto immunodeficiency disease (AIDS) related neoplasm Kaposi sarcoma in Iraqi man: a case report
}

\author{
Haider Saadoon Q. Alhelfi ${ }^{1 *}$, Rasha Khalil A. Alsaad ${ }^{2}$, Ahmed Salih H. Alshewered ${ }^{3}$ \\ ${ }^{1}$ M.B. Ch.B., F.I.B.M.S; Oncologist; Department of Medicine, College of Medicine, University of Missan; Missan, Iraq \\ ${ }^{2}$ M.Sc. Parasitology; Lecturer; College of Veterinary Medicine, University of Basrah; Basrah, Iraq \\ ${ }^{3}$ M.B. Ch.B.; Permanent radiotherapy doctor; Missan Radiotherapy Center; Missan, Iraq \\ *Corresponding author E-mail:masterr78@ymail.com
}

\begin{abstract}
The aim of this article is to report unusual case of KS presenting in the patient with negative serology for AIDS. Kaposi Sarcoma (KS) is a rare entity. It is a vascular neoplasm mainly affecting the skin of the lower extremities. Although it is the most common neoplasm affecting patients with AIDS, sporadic cases in HIV-negative people have been reported but it is rare. Here, we present a case of a nonauto immunodeficiency disease (AIDS) related KS. A 72 year-old male, Iraqi patient presented to our oncology outpatient's unit in AlShafaa Oncology Center with multiple subcutaneous nodules. The diagnosis was based on histopathological and immunohistochemical analysis of the excised lesion, serology tests and imaging investigations. The patient had a 13-month history of a slowly progressing pigmented multiple lesions on the lower and upper extremities. The patient had visceromegaly as splenomegaly at the time of presentation. His serum was negative for the common types of viral infections including Human Immunodeficiency Virus (HIV) on routine serology. On general examination, the man look well, no pallor, no fever, no cachexia present and no jaundice. There are a multiple defined, irregular hyper-pigmented nodules to plaques were present on forearm, legs, feet and hands. The nodules and plaques were firm in consistency and was non-tender. The lesions were deep reddish to brownish in color. The size of the lesions were arranged from $0.5-1.5 \mathrm{~cm}$. No scaling was evident. The patient was under treatment with chemotherapeutic regimen of Paclitaxel.
\end{abstract}

Keywords: AIDS, immunohistochemistry, HIV, Kaposi, Sarcoma.

\section{Introduction}

Kaposi Sarcoma (KS) was first described in 1872 by Dr. Moritz Kaposi (1837-1902), a Hungarian dermatologist initially termed "idiopathic multiple pigmented sarcoma" and later recognized in the literature as (classical) Kaposi's sarcoma (Sanna et al. 2000, Mohanna et al. 2007 \& Gilani et al. 2009). It is a malignancy of skin, mucous membranes and blood vessels (Gilani et al. 2009). It has a known association with Human Immunodeficiency Virus (HIV) infection (Sanna et al. 2000 \& Mohanna et al. 2006). It was the first clue to the diagnosis of Acquired Immunodeficiency Syndrome (AIDS) in the late 1970's (Beral et al. 1990). HHV-8 infection is required for the development of classical KS, but not all infected persons develop the disease. Four variants of are known that is classical (sporadic), endemic (African), iatrogenic (immunosuppressive drugs associated) and AIDS-associated (Mohanna et al. 2006 \& Solivetti et al. 2011). Classic KS occurs sporadically and is seen in immunocompetent individuals and may present as bluish-red or hyper-pigmented papules, plaques or nodules over feet or hands with pedal edema (Mohanna et al. 2006 \& Mohanna et al. 2007). The incidence is higher in Southern and Eastern European countries and the condition is more commonly found in Jewish, Italian and Greek populations (Dal Maso et al. 2005). It is more common in male than female having ratio of $15: 1$ with predilection for elderly males (Mohanna et al. 2006). Current treatment for classical Kaposi's sarcoma is based on radiation therapy, while chemotherapy has only a marginal role (Mohanna et al. 2006, Mohanna et al. 2007 \& Solivetti et al. 2011).

\section{Case presentation}

a) History and clinical examination

A 72 year-old male, self-employer Iraqi patient presented to our oncology outpatient's unit in Al-Shafaa Oncology Center with multiple hard subcutaneous nodules. The worst affected skin areas given in order of severity of involvement were the legs, feet, forearms and hands. The patient had a 13-month history of a slowly progressing pigmented multiple lesions on the lower and upper extremities. On general examination, the man look well, no pallor, no fever, no cachexia present and no jaundice. The malaise, fatigue and fever were all absent. In local examination there are a multiple violaceous defined, irregular hyper-pigmented nodules to plaques were present on forearm, legs, feet and hands. The nodules and plaques were firm in consistency, non-pruritic, non-scaly and non-tender. The lesions were deep reddish to brownish in colour, see figures (1, 2, 3 and 4). The patient was married and have 7 off-springs. His blood group is AB positive. The patient belonged to a poor socioeconomic class.

b) Laboratory investigations

The routine medical tests', i.e. Complete Blood Picture (CBC), hepatic and renal functional profiles were found within normal limits. 


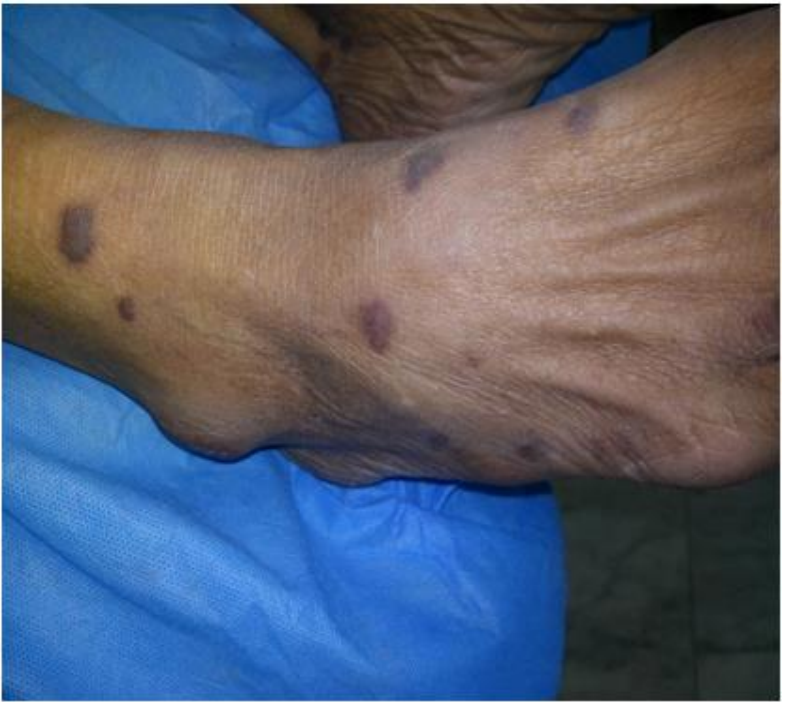

Fig. 1: Shows Multiple Ill Defined, Irregular Hyper-Pigmented Nodules in Dorsum Aspect of Right Foot.



Fig. 2: Shows Multiple Ill Defined, Irregular Hyper-Pigmented Nodules in Extensor Aspect of the Left Hand.

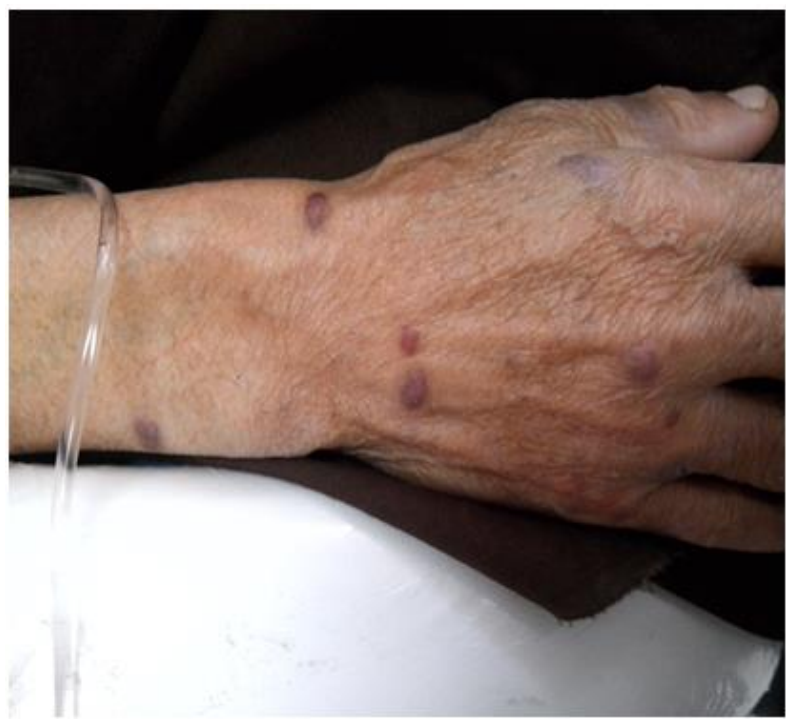

Fig. 3: Shows Multiple Ill Defined, Irregular Hyper-Pigmented Nodules in Extensor Aspect of Right Forearm and Hand.

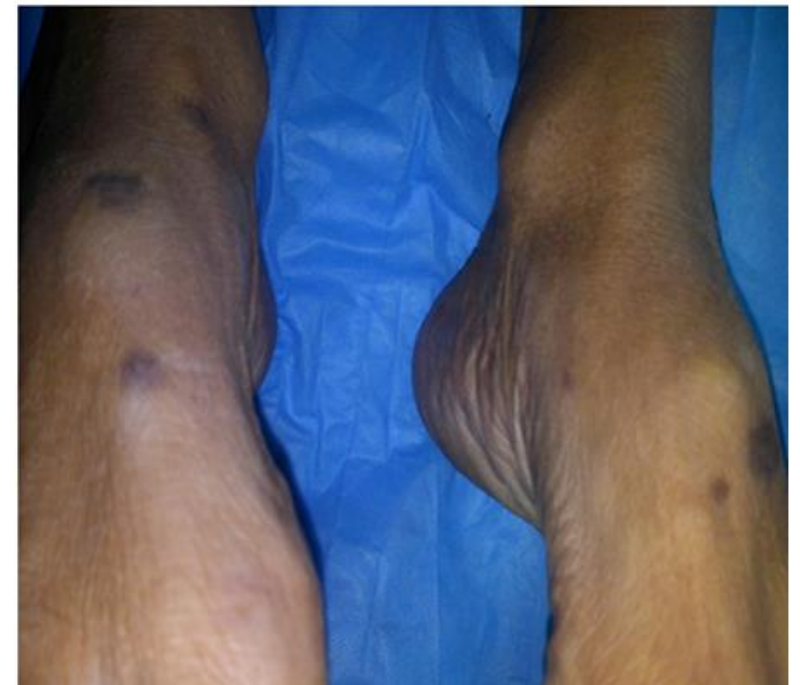

Fig. 4: Shows Multiple Ill Defined, Irregular Hyper-Pigmented Nodules in Dorsum Aspect of Both Feet.

c) Histopathology

The histopathology report was typical of Kaposi sarcoma with involvement of lymphatic channels. The biopsy showen the classical histology of KS, that is, a proliferation of spindle cells among bizarre shaped blood vessels and extravasation red blood cells.

d) Imaging

Chest radiograph and ultrasonography scan of an abdomen were normal despite one finding which is enlarge spleen. The thoracic and abdominal computed tomography $\mathrm{CT}$ scan, were all negative for the presence of disease despite two findings in the abdominal CT scan, which are a large spleen of $21 \mathrm{~cm}$ and single enlarge lymph node at para aortic region, see figures $(5,6,7$ and 8$)$.

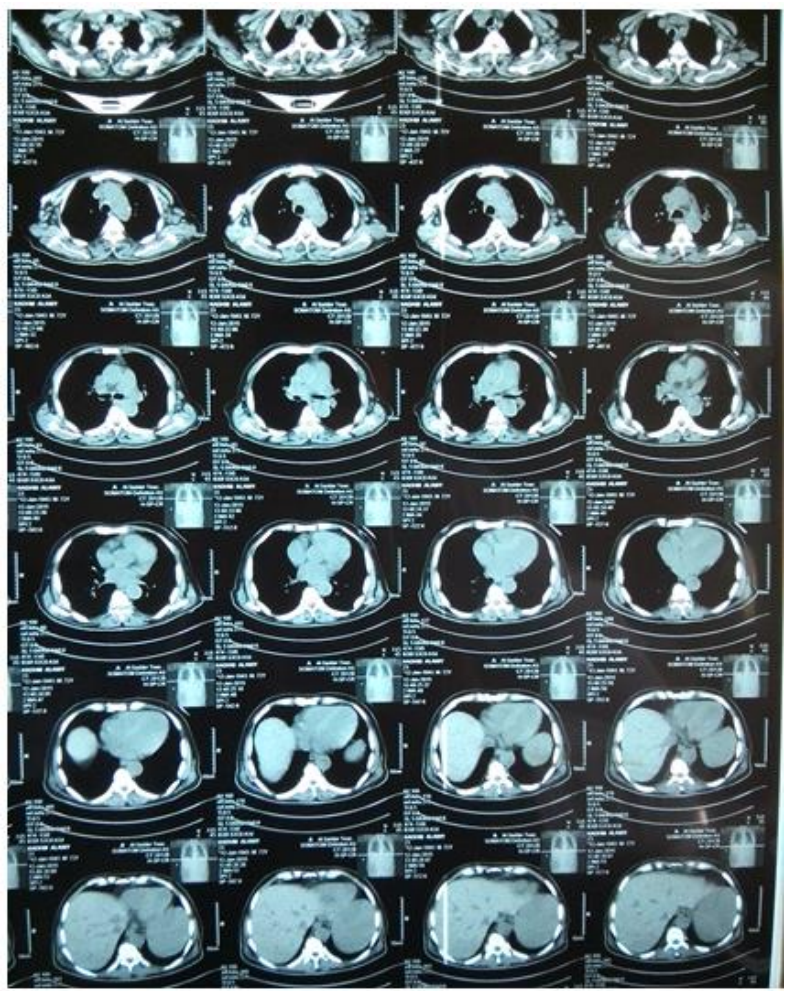

Fig. 5: Abdominal Computed Tomography CT scan. 


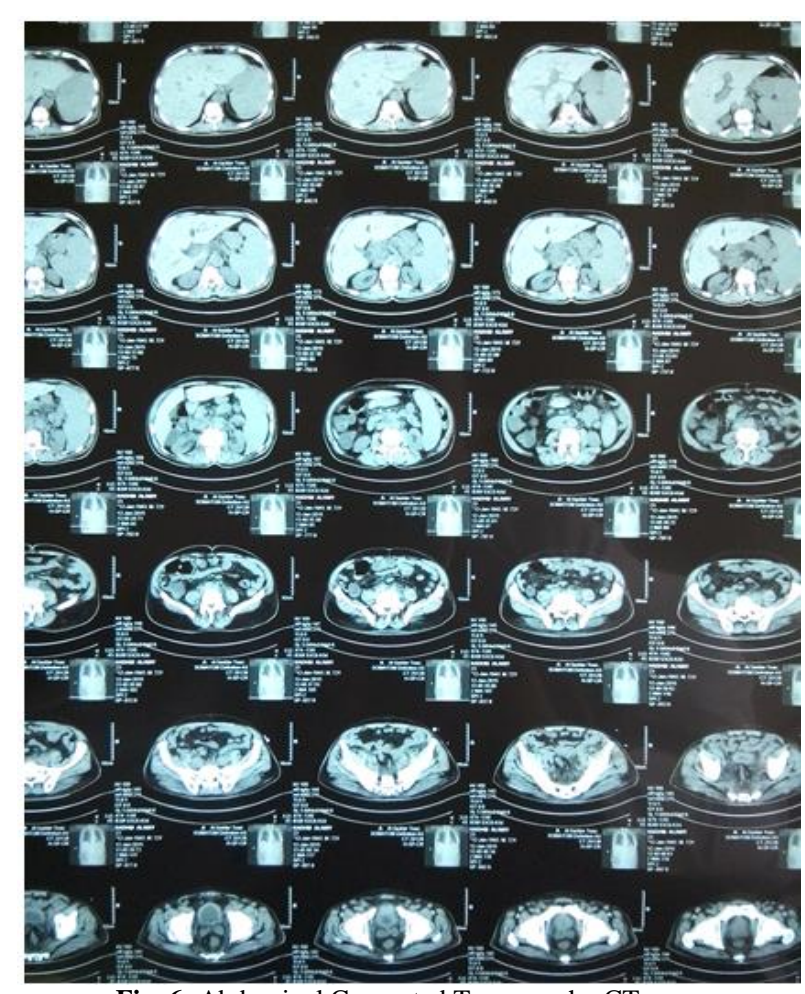

Fig. 6: Abdominal Computed Tomography CT scan.

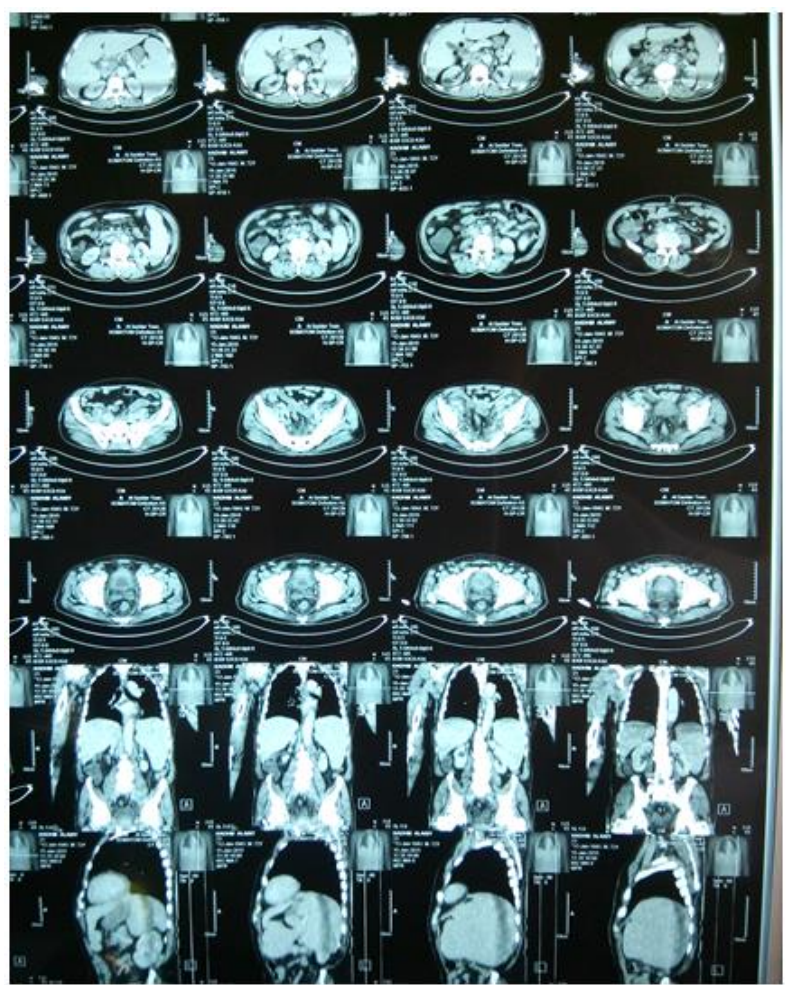

Fig. 7: Chest \& Abdominal Computed Tomography CT scan

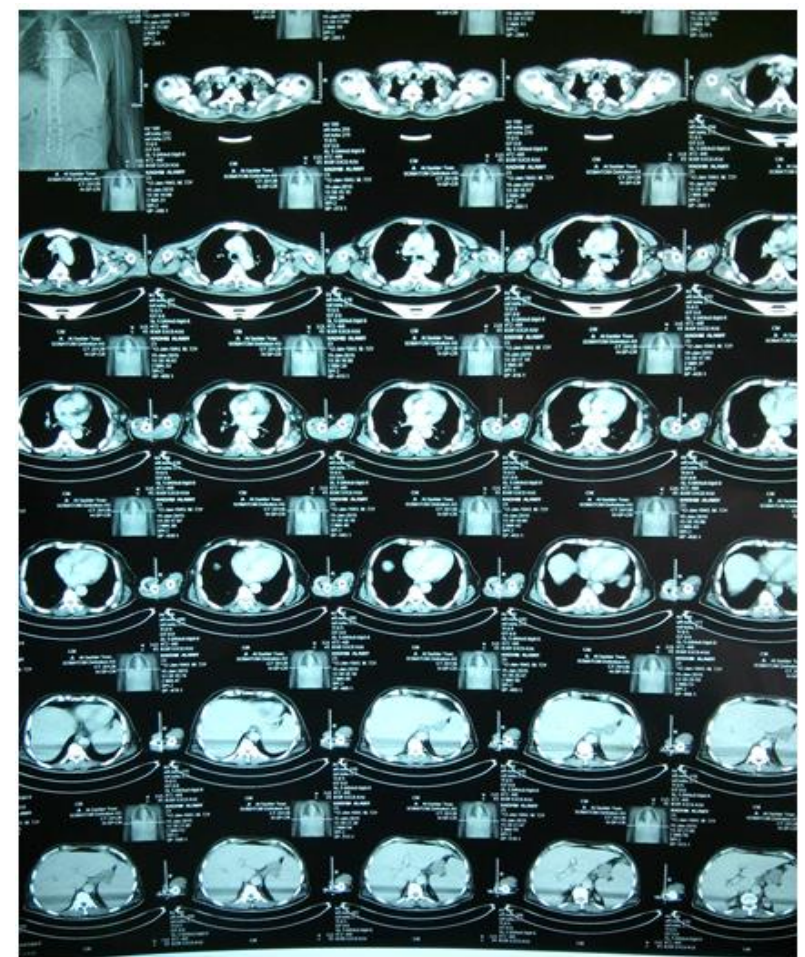

Fig. 8: Chest \& Abdominal Computed Tomography CT scan.

e) Serology

Serology for analytical cytometry and his CD-4/CD-8 counts were analysed and were found to be within the normal range so the testing for HIV infection was negative. The HBs Ag and VDRL were negative. Because of lack of facility's Polymerase chain reaction (PCR) for human herpesvirus 8 (HHV-8) could not be done. Sputum for Acid-Fast Bacilli (AFB) and Mantoux test were negative.

f) IHC immunohistochemistry

The results of immune-staining for the other markers were positively stained for tumor makers such as CD31, CD34, D2-40 and vimentin, which supported their diagnosis. SMA, S-100, bcl-2 and p53 were totally negative.

\section{Discussion}

Our case reported is a 72 year-old male, Iraqi patient presented to our oncology outpatient's unit with multiple hard subcutaneous nodules. The worst affected skin areas given in order of severity of involvement were the legs, feet, forearms and hands. Local examination there are a multiple ill defined, irregular hyper-pigmented nodules to plaques were present on forearm, legs, feet and hands. The nodules and plaques were firm in consistency, non-pruritic, non-scaly and non-tender. The lesions were deep reddish to brownish in color. Kaposi Sarcoma (KS), an opportunistic sequel of HIV infection, is considered as the common AIDS associated neoplasm (Beral et al. 1990). Non-HIV Kaposi sarcoma is a rare entity in this part of the world and clinicians are mostly not familiar with its presentation. It most often arises in middle-aged and elderly men of Mediterranean or Jewish descent (Freidman-Kien et al. 1982). Extensive and multifocal skin involvement by pinkish red macules and papules of variable sizes and no laboratory evidence of HIV infection positively were the main features of the KS variant in this region (Gilani et al. 2009). It usually presents as multiple firm purple blue or reddish-brown plaques and nodules typically appearing over hands and feet and progress up to arms and thighs (Sanjay et al. 2014). In 10\% of cases visceral or mucosal involvement is seen (Sanjay et al. 2014).

Diagnosis is made by clinical and histopathological correlation. The different histological patterns have been described and classified into six major groups: inflammatory, granulomatous, predominantly angiomatous, predominantly spindle cell, mixed and ana- 
plastic cell pattern with strong variations inside the same tumor (Bisceglia et al. 1991). Serology for analytical cytometry and his CD-4/CD-8 counts were analyzed and were found to be within normal range so the testing for HIV infection was negative. CD4/CD-8 ratio was done which favoured a non-HIV origin. A large body of evidence has strongly linked all KS clinical variants with HHV8 infection (Antman et al. 2000). The presence of HHV8 infection is now considered essential for the development of KS (Antman et al. 2000 \& Vitale et al. 2000). A sensitive method for detecting HHV8 in the mononuclear cells of individuals who subsequently develop KS is PCR. PCR is considered to be a specific and sensitive means of verifying KS in the differential diagnosis of angioproliferative lesions (Whitby et al. 1995 \& Dictor et al. 1996). But because of lack of facilities Polymerase chain reaction (PCR) for human herpesvirus 8 (HHV-8) could not be done. In non-AIDS related KS, ABV chemotherapy has documented evidence of efficacy. Treatment depends on extent and localization of lesions. It includes non-intervention, cryoablation, surgical excision, laser therapy. Liposomal Dounoblastine, Paclitaxil has also been used in KS (Gill et al. 1996). The most active cytotoxic agents include vinblastine, vincristine, bleomycin, etoposide, doxorubicin, daunorubicin and paclitaxel (Shetty et al. 2005). The indications for systemic chemo therapy are (Fatahzadeh 2012):

- Symptomatic visceral or mucosal involvement.

- Diffuse symptomatic lesions on multiple body parts that are not easily encompassed in a single or a limited number of radiation fields.

- Extensive nodular disease or diffuse involvement of a large portion of an extremity.

- Bulky disease in a localized area of one limb that cannot be encompassed within a single radiation therapy port.

- Moderate to severe associated lymphedema beyond what can be controlled with elastic stockings.

Radiation therapy was again effective, achieving a partial remission of the cutaneous lesions with pain control. Local radiation is today the treatment of choice for small and localized lesions (Miles et al. $1997 \&$ Brenner et al. 1999), producing in most cases long-lasting remissions and optimal local control.

So the patient was under treatment with chemotherapeutic regimen of Paclitaxel $120 \mathrm{mg} / \mathrm{m}^{2}$ every 21 days for 6 cycles.

Standardized staging criteria help when deciding on appropriate local or systemic therapy and for assessing and comparing response therapies.

\section{Conclusion}

Kaposi's sarcoma is a rare neoplasm of elderly residents of the Mediterranean area. Skin biopsy is important for making the correct diagnosis, with the added use of immunohistochemistry. Kaposi's sarcoma is characterized by an indolent clinical course and a good prognosis. Mono-chemotherapy give a good response in treatment of KS. Radiation therapy is still the treatment of choice for small and localized lesions, producing long-lasting remissions.

\section{References}

[1] Antman K \& Chang Y (2000). Kaposi's sarcoma. N Engl J Med, 342, 1027-1038. http://dx.doi.org/10.1056/NEJM200004063421407.

[2] Beral V, Peterman TA, Berkelman RL \& Jaffer HW (1990). Kaposi sarcoma among persons with AIDS: a sexually trans-mitted infection. Lancet, 335, 123-128. http://dx.doi.org/10.1016/0140-6736(90)90001L.

[3] Brenner B, Rakowsky E, Katz A, Gutman H, Sulkes A \& Schacter J (1999). Tailoring treatment for classical Kaposi's sarcoma: comprehensive clinical guidelines. Int J Oncol, 14, 1097-1102. http://dx.doi.org/10.3892/ijo.14.6.1097.

[4] Bisceglia M, Bosman C, Carlesimo OA, Innocenzi D \& Quirke P (1991). Kaposi's sarcoma: a clinico-pathologic overview. Tumori, 77, 291-310.
[5] Dal Maso L, Polesel J, Ascoli V, Zambon P, Budroni M \& Fer-retti S (2005). Classic Kaposi's sarcoma in Italy, 1985-1998. Br J Cancer, 92, 188-193. http://dx.doi.org/10.1038/sj.bjc.6602265.

[6] Dictor M, Rambech E, Way D, Witte M \& Bendsoe N (1996). Human herpesvirus 8 (Kaposi's sarcoma-associated herpesvirus) DNA in Kaposi's sarcoma lesions, AIDS Kaposi's sarcoma cell lines, endothelial Kaposi's sarcoma simulators, and the skin of immunosuppressed patients. Am J Pathol, 148, 2009-2016.

[7] Fatahzadeh M (2012). Kaposi sarcoma: review and medical management update. Oral Surg Oral Med Oral Pathol Oral Radiol, 113(1), 216.

[8] Freidman-Kien AE, Laubenstein LJ, Rubinstein P, Buimovici-Klein E, Marmoa M \& Stahl R (1982). Disseminated Kaposi sarcoma in young homosexual men. Ann Intern Med, 96, 693-700. http://dx.doi.org/10.7326/0003-4819-96-6-693.

[9] Gilani JA, Khan AU, Shahid S, Khan IU \& Khan SU (2009). Clinical Presentation of Non-HIV Kaposi Sarcoma. Journal of the College of Physicians and Surgeons Pakistan, 19(9), 586-588.

[10]Gill PS, Wernz J \& Scaden DT (1996). Randomized phase trial of liposomal Daunoblastine versus Doxorubicin, Bleomysin and Vincristine in AIDS related Kaposi sarcoma. J Clin Oncol, 14, 2353

[11]Miles SA, Mitsuyasu RI \& Aboulafia DM (1997). Kaposi's sarcoma. In: De Vita V, Hellman S, Rosenberg SA, eds. Principles and Practice of Oncology. 5th ed. Philadelphia: Lippincott-Raven Publishers; p. 2445-2449.

[12]Mohanna S, Bravo F, Ferrufino JC, Sanchez J \& Gotuzzo E (2007). Classic Kaposi's sarcoma presenting in the oral cavity of two HIVnegative Quechua patients. Med Oral Patol Oral Cir Bucal, 12, 365368.

[13]Mohanna S, Maco V, Gown A, Morales D, Bravo F \& Gotuzzo E (2006). Is classic Kaposi's sarcoma endemic in Peru: report of a case in an indigenous patient. Am J Trop Med Hyg, 75, 324-326.

[14] Sanjay NA, Anuprita AR \& Subodhkumar DJ (2014). Classic Kaposi's sarcoma: a rare case with unusual presentation. Our Dermatol Online, 5(1), 68-70. http://dx.doi.org/10.7241/ourd.20141.18.

[15] Sanna P, Rosselli M, Mainetti C, Gilliet F, Sessa C, Bernier J \& Cavalli F (2000). Classical (HIV-negative) cutaneous Kaposi's sarcoma: a case report and a short review of the literature. Schweiz Med Wochenschr, 130, 988-992.

[16] Shetty K (2005). Management of oral Kaposi's sarcoma lesions on HIV-positive patient using highly active antiretroviral thera-py: Case report and a review of the literature. Oral Oncol, 41, 226-229. http://dx.doi.org/10.1016/i.ooe.2005.06.006.

[17] Solivetti FM, Elia F, Latini A, Cota C, Cordiali-Fei P \& Di Carlo A (2011). AIDS-Kaposi Sarcoma and Classic Kaposi Sarcoma: are different ultrasound patterns related to different variants. J Exp Clin Cancer Res, 30, 40. http://dx.doi.org/10.1186/1756-9966-30-40.

[18] Vitale F, Viviano E, Perna AM, Bonura F, Mazzola G, Ajello F \& Romano N (2000). Serological and virological evidence of non-sexual transmission of human herpesvirus type 8 (HHV8). Epidemiol Infect, 125, 671-675. http://dx.doi.org/10.1017/S0950268800004726.

[19] Whitby D, Howard MR, Tenant-Flowers M, Brink NS, Copas A, Boshoff C, Hatzioannou T, Suqqett FE, Aldam DM \& Denton AS (1995). Detection of Kaposi sarcoma associated herpesvirus in peripheral blood of HIV-infected individuals and progression to Kaposi's sarcoma. Lancet, 346, 799-802. http://dx.doi.org/10.1016/S01406736(95)91619-9. 